\title{
STABILITY OF LAMINATED BEAMS WITH SECOND SOUND
}

\author{
C. A. RAPOSO ${ }^{1, *}$, O. P. VERA VILLAGRAN ${ }^{2}$, C. A. S. NONATO $^{3}$, H.H. NGUYEN ${ }^{4}$ \\ ${ }^{1}$ Department of Mathematics, Federal University of São João del-Rei, Brazil \\ ${ }^{2}$ Department of Mathematics, Universidad del Bío-Bío, Chile \\ ${ }^{3}$ Department of Mathematics, Federal University of Bahia, Brazil \\ ${ }^{4}$ LMAP/UMR E2S-UPPA CNRS 5142, France \\ *Corresponding author: raposo@ufsj.edu.br
}

Received Feb. 8, 2019

\begin{abstract}
Aвstract. In this manuscript, we prove the well-posedness and exponential stability for a thermoelastic structure given by a laminated Timoshenko beam model consisting of two identical layers, taking into account that an adhesive of the small thickness is bonding these layers and produce a interfacial slip. We consider the action of the temperature difference and heat flux as stabilization mechanisms. We use semigroup approach, Lumer-Phillips theorem for existence and uniqueness of solution and Gearhart-Huang-Prüss theorem to prove the exponential stability.
\end{abstract}

2010 Mathematics Subject Classification. 35B40; 35L53; 74F05; 74F20.

Key words and phrases. thermoelasticity; Cattaneo's law; laminated beam.

\section{INTRODUCTION}

The two-layered beam with structural damping is given by the following system

$$
\begin{array}{r}
\rho u_{t t}+G\left(\psi-u_{x}\right)_{x}=0, \\
I_{\rho}\left(3 S_{t t}-\psi_{t t}\right)-G\left(\psi-u_{x}\right)-D\left(3 S_{x x}-\psi_{x x}\right)=0, \\
3 I_{\rho} S_{t t}+3 G\left(\psi-u_{x}\right)+4 \delta_{0} S+4 \gamma_{0} S_{t}-3 D S_{x x}=0,
\end{array}
$$

where $(x, t) \in(0, L) \times(0, \infty)$.

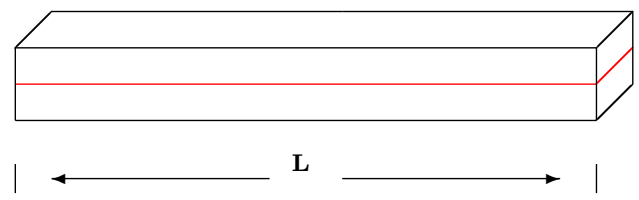

The model (1.1)-(1.3) was derived under assumption of the Timoshenko theory in [10], where $u(x, t)$ denotes the transverse displacement, $\psi(x, t)$ represents the rotation angle and $S(x, t)$ is proportional to the amount of slip along the interface at time $t$ and longitudinal spatial variable $x$, respectively, and $\rho, G, I_{\rho}, D, \delta_{0}, \gamma_{0}$ are

DOI: $10.28924 / \mathrm{APJM} / 7-5$ 
the density of the beams, the shear stiffness, mass moment of inertia, flexural rigidity, adhesive stiffness and adhesive damping of the beams. From now and on, considering $s(x, t)=3 S(x, t), \rho_{1}=\rho, \rho_{2}=I_{\rho}, k=G$, $b=D, 3 \delta=\delta_{0}, 3 \gamma=\gamma_{0}$, we deduce from (1.1)-(1.3) the following system

$$
\begin{aligned}
\rho_{1} u_{t t}+k\left(\psi-u_{x}\right)_{x} & =0, \\
\rho_{2}(s-\psi)_{t t}-b(s-\psi)_{x x}-k\left(\psi-u_{x}\right) & =0, \\
\rho_{2} s_{t t}-b s_{x x}+3 k\left(\psi-u_{x}\right)+4 \delta s+4 \gamma s_{t} & =0 .
\end{aligned}
$$

Combining (1.5) and (1.6) we have

$$
\begin{aligned}
\rho_{1} u_{t t}+k\left(\psi-u_{x}\right)_{x} & =0, \\
\rho_{2} \psi_{t t}-b \psi_{x x}+4 k\left(\psi-u_{x}\right)+4 \delta s+4 \gamma s_{t} & =0 .
\end{aligned}
$$

Note that for $s=0$ in (1.8) we obtain the conservative Timoshenko system. There is an extensive bibliography for the thermoelastic Timoshenko system and thermoelasticity with Cattaneo's law (second sound). We cite for instance $[3,4,18,19,21,26,30-32]$. For laminated beam, there are only a few papers specifically in connection with the system (1.1)-(1.3). Among them, we mention the references [6,9,15,16,27,33]. More recently, dynamics of laminated Timoshenko beams were studied in [7] where the authors established the existence of smooth finite dimensional global attractors for the corresponding solution semigroup. Hybrid laminated Timoshenko beam model was considered in [28] where the beam is fastened securely on the left while on the right it is free and has an attached container. Using the semigroup approach and a result of A. Borichev and Y. Tomilov (see [5]), the authors proved that the solution is polynomially stable.

In [33], the authors proved that the frictional damping created by the interfacial slip alone is not enough to stabilize the system (1.1)-(1.3) exponentially to its equilibrium state, then another mechanism of stabilization is necessary to be introduced to produce exponential decay of solution. With this approach, inspired by the result [29], the exponential stability for a structure with interfacial slip taking into account the frictional damping was proved in [27].

In the theory of thermoelasticity of second sound, the heat equation is of hyperbolic type and so predicts a finite speed of heat propagation. See $[19,26,32]$ for more detail on this theory. About thermoelastic Timoshenko system in the presence of the frictional damping and heat conduction modelled by Cattaneo's law for the system bellow

$$
\begin{array}{r}
\rho_{1} \varphi_{t t}-\sigma\left(\varphi_{x}+\psi\right)_{x}+\mu \varphi_{t}=0, \\
\rho_{2} \psi_{t t}-b \psi_{x x}+k\left(\varphi_{x}+\psi\right)+\beta \theta_{x}=0, \\
\rho_{3} \theta_{t}+\gamma q_{x}+\delta \psi_{t x}=0, \\
\tau_{0} q_{t}+q+\kappa \theta_{x}=0,
\end{array}
$$

several exponential decay results for both linear and nonlinear cases have been established in $[18,19]$.

In the absence of the frictional damping in the work [31], it was shown that the coupling via Cattaneo's law causes loss of the exponential decay usually obtained in the case of coupling via Fourier's law. The authors 
considered the following system

$$
\begin{aligned}
\rho_{1} \varphi_{t t}-k\left(\varphi_{x}+\psi\right)_{x} & =0, \\
\rho_{2} \varphi_{t t}-b \psi_{x x}+k\left(\varphi_{x}+\psi\right)+\delta \theta_{x} & =0, \\
\rho_{3} \theta_{t}+q_{x}+\delta \psi_{x t} & =0, \\
\tau q_{t}+\beta q+\theta_{x} & =0 .
\end{aligned}
$$

Later, in [3] the stability number $\chi_{0}$ given by

$$
\chi_{0}=\left(\tau-\frac{\rho_{1}}{k \rho_{3}}\right)\left(\rho_{2}-\frac{b \rho_{1}}{k}\right)-\frac{\tau \rho_{1} \delta^{2}}{k \rho_{3}}
$$

was introduced and the authors proved that for (1.9), the exponential stability holds if and only if $\chi_{0}=0$, that is, the velocities of waves propagations are equal. In fact, for all the literature concerning Timoshenko system, when only one dissipation is taking into account the conclusion is that the exponential stability holds if and only if the velocities of waves propagations are equal.

Well-posedness and asymptotic behaviour for a laminated beam system in thermoelasticity with Fourier's law and Cattaneo's law forms the centre of this work. The main difficulty carrying out this paper is the presence of Fourier's law of heat conduction that can produce lack of exponential stability when the wave speed is different for systems partially damped, see [22]. Recent investigations lead that the existence of a second spectrum is an essential element to justify from the physical point of view the imposed condition of equal wave speed. For more information on this subject, we cite [1] and reference therein.

However, if the damping terms are added in all equations (as in $[27,29]$ ), the energy of the system decays exponentially without assumption over the coefficients of the system. Motivated by the above results, we will investigate the damped system below, that is, we are interested in the asymptotic behaviour of a thermoelastic structure with interfacial slip and second sound derived from (1.1)-(1.3). More precisely, we deal with the system as follows

$$
\begin{aligned}
\rho_{1} u_{t t}+k\left(\psi-u_{x}\right)_{x} & =0, \\
\rho_{2}(s-\psi)_{t t}-b(s-\psi)_{x x}-k\left(\psi-u_{x}\right)+\beta(s-\psi)_{t}+\mu \theta_{x} & =0, \\
\rho_{2} s_{t t}-b s_{x x}+3 k\left(\psi-u_{x}\right)+4 \delta s+4 \gamma s_{t} & =0, \\
\theta_{t}-\theta_{x x}+\nu q_{x}+\mu(s-\psi)_{x t} & =0, \\
q_{t}+q+\nu \theta_{x} & =0,
\end{aligned}
$$

where $(x, t) \in(0, L) \times(0, \infty)$ with Dirichlet boundary conditions

$$
\begin{gathered}
u(0, t)=\psi(0, t)=s(0, t)=\theta(0, t)=q(0, t)=0, \\
u(L, t)=\psi(L, t)=s(L, t)=\theta(L, t)=q(L, t)=0
\end{gathered}
$$

and initial data

$$
\begin{aligned}
(u(x, 0), \psi(x, 0), s(x, 0), \theta(x, 0), q(x, 0)) & =\left(u_{0}(x), \psi_{0}(x), s_{0}(x), \theta_{0}(x), q_{0}(x)\right), \\
\left(u_{t}(x, 0), \psi_{t}(x, 0), s_{t}(x, 0), \theta_{t}(x, 0), q_{t}(x, 0)\right) & =\left(u_{1}(x), \psi_{1}(x), s_{1}(x), \theta_{1}(x), q_{1}(x)\right) .
\end{aligned}
$$


In this system, $u=u(x, t)$ is the transversal displacement, $\psi=\psi(x, t)$ represents the rotation angle, $s=s(x, t)$ is proportional to the amount of slip along the interface, $\theta=\theta(x, t)$ is the temperature difference, $q=q(x, t)$ is the heat flux and $\rho_{1}, \rho_{2}, k, b, \delta, \beta, \gamma, \mu, \nu$ are positive constants.

In order to achieve our goal, we use the Sobolev spaces and semigroup theory with its properties as in $[2,24]$. For asymptotic behaviour, the idea presented by Liu and Zheng [17] for dissipative systems is used. This technique is different from some others in the literature, like as the traditional energy method, see Rivera [20], the direct method, see Kormonik [13,14] and Nakao's method, see [23]. This manuscript is organized as follows. In Section 2, we deal with setting of the semigroup, where we prove the well-posedness of the problem. In Section 3, we show the exponential stability using the result obtained by Gearhart [8] and Huang [11] independently (see also Prüss [25]).

\section{Setting of the Semigroup}

In this section, for convenience, we introduce another variables $\xi$ and $z$ given by

$$
\xi=s-\psi
$$

Then (1.10) change to

$$
\begin{aligned}
\rho_{1} u_{t t}+k\left(s-\xi-u_{x}\right)_{x} & =0, \\
\rho_{2} \xi_{t t}-b \xi_{x x}-k\left(s-\xi-u_{x}\right)+\beta \xi_{t}+\mu \theta_{x} & =0, \\
\rho_{2} s_{t t}-b s_{x x}+3 k\left(s-\xi-u_{x}\right)+4 \delta s+4 \gamma s_{t} & =0, \\
\theta_{t}-\theta_{x x}+\nu q_{x}+\mu \xi_{x t} & =0, \\
q_{t}+q+\nu \theta_{x} & =0,
\end{aligned}
$$

where $(x, t) \in(0, L) \times(0, \infty)$ with boundary conditions

$$
\begin{gathered}
u(0, t)=\xi(0, t)=s(0, t)=\theta(0, t)=q(0, t)=0, \\
u(L, t)=\xi(L, t)=s(L, t)=\theta(L, t)=q(L, t)=0
\end{gathered}
$$

and initial data

$$
\begin{aligned}
(u(x, 0), \xi(x, 0), s(x, 0), \theta(x, 0), q(x, 0)) & =\left(u_{0}(x), \xi_{0}(x), s_{0}(x), \theta_{0}(x), q_{0}(x)\right), \\
\left(u_{t}(x, 0), \xi_{t}(x, 0), s_{t}(x, 0), \theta_{t}(x, 0), q_{t}(x, 0)\right) & =\left(u_{1}(x), \xi_{1}(x), s_{1}(x), \theta_{1}(x), q_{1}(x)\right) .
\end{aligned}
$$

For the standard $L^{2}(0, L)$ space, the scalar product and the norm are denoted by

$$
\langle\varphi, \psi\rangle_{L^{2}(0, L)}=\int_{0}^{L} \varphi \psi d x, \quad\|\psi\|_{L^{2}(0, L)}^{2}=\int_{0}^{L}|\psi|^{2} d x .
$$

Introducing the vector function $U=\left(u, u_{t}, \xi, \xi_{t}, s, s_{t}, \theta, q\right)^{T}$, the system (2.1)-(2.7) can be written as

$$
\left\{\begin{aligned}
U_{t}-\mathcal{A} U & =0 \\
U(0) & =U_{0}=\left(u_{0}, u_{1}, \xi_{0}, \xi_{1}, s_{0}, s_{1}, \theta_{0}, q_{0}\right)^{T},
\end{aligned}\right.
$$


where the operator $\mathcal{A}: \mathcal{D}(\mathcal{A}) \subset \mathcal{H} \rightarrow \mathcal{H}$ is defined by

$$
\mathcal{A}\left(\begin{array}{c}
u \\
u_{t} \\
\xi \\
\xi_{t} \\
s \\
s_{t} \\
\theta \\
q
\end{array}\right)=\left(\begin{array}{c}
u_{t} \\
-\frac{k}{\rho_{1}}\left(s-\xi-u_{x}\right)_{x} \\
\xi_{t} \\
\frac{1}{\rho_{2}}\left[b \xi_{x x}+k\left(s-\xi-u_{x}\right)-\beta \xi_{t}-\mu \theta_{x}\right] \\
s_{t} \\
\frac{1}{\rho_{2}}\left[b s_{x x}-3 k\left(s-\xi-u_{x}\right)-4 \delta s-4 \gamma s_{t}\right] \\
\theta_{x x}-\nu q_{x}-\mu \xi_{x t} \\
-q-\nu \theta_{x}
\end{array}\right)
$$

where the space $\mathcal{H}$ is given by

$$
\mathcal{H}=\left[H_{0}^{1}(0, L) \times L^{2}(0, L)\right]^{3} \times H_{0}^{1}(0, L)^{2}
$$

is a Hilbert space equipped with the inner product given by

$$
\begin{aligned}
\langle U, \bar{U}\rangle_{\mathcal{H}}= & 3 \rho_{1} \int_{0}^{L} u_{t} \bar{u}_{t} d x+3 k \int_{0}^{L}\left(s-\xi-u_{x}\right)\left(\bar{s}-\bar{\xi}-\bar{u}_{x}\right) d x \\
& +\rho_{2} \int_{0}^{L} s_{t} \bar{s}_{t} d x+b \int_{0}^{L} s_{x} \bar{s}_{x} d x+4 \delta \int_{0}^{L} s \bar{s} d x+3 \int_{0}^{L} \theta \bar{\theta} d x \\
& +3 \int_{0}^{L} q \bar{q} d x+3 \rho_{2} \int_{0}^{L} \xi_{t} \bar{\xi}_{t} d x+3 b \int_{0}^{L} \xi_{x} \bar{\xi}_{x} d x
\end{aligned}
$$

and the correspondent norm is

$$
\begin{aligned}
\|U\|_{\mathcal{H}}^{2}= & 3 \rho_{1} \int_{0}^{L}\left|u_{t}\right|^{2} d x+3 k \int_{0}^{L}\left|s-\xi-u_{x}\right|^{2} d x+\rho_{2} \int_{0}^{L}\left|s_{t}\right|^{2} d x+b \int_{0}^{L}\left|s_{x}\right|^{2} d x \\
& +4 \delta \int_{0}^{L}|s|^{2} d x+3 \int_{0}^{L}|\theta|^{2} d x+3 \int_{0}^{L}|q|^{2} d x+3 \rho_{2} \int_{0}^{L}\left|\xi_{t}\right|^{2} d x+3 b \int_{0}^{L}\left|\xi_{x}\right|^{2} d x .
\end{aligned}
$$

Denoting $\Lambda=\left(u, u_{t}, \xi, \xi_{t}, s, s_{t}, \theta, q\right)^{T}$, the domain of $\mathcal{A}$ is given by

$$
\mathcal{D}(\mathcal{A})=\left\{\Lambda \in \mathcal{H} ; \quad u, \xi, s, \theta \in H^{2}(0, L) \cap H_{0}^{1}(0, L), \quad u_{t}, \xi_{t}, s_{t}, q \in H_{0}^{1}(0, L)\right\} .
$$

Note that $\mathcal{D}(\mathcal{A})$ is independent of time $t>0$ and clearly, $\mathcal{D}(\mathcal{A})$ is dense in $\mathcal{H}$. Next, will prove that the operator $\mathcal{A}$ is dissipative.

Proposition 2.1. For $U=\left(u, u_{t}, \xi, \xi_{t}, s, s_{t}, \theta, q\right)^{T} \in D(\mathcal{A})$ we have

$$
\langle\mathcal{A} U, U\rangle_{\mathcal{H}}=-4 \gamma \int_{0}^{L}\left|s_{t}\right|^{2} d x-3 \int_{0}^{L}\left|\theta_{x}\right|^{2} d x-3 \int_{0}^{L}|q|^{2} d x-3 \beta \int_{0}^{L}\left|\xi_{t}\right|^{2} d x \leq 0 .
$$

Proof. Direct computation, using (2.10), gives

$$
\begin{aligned}
\langle\mathcal{A} U, U\rangle_{\mathcal{H}}= & -3 k\left\langle\left(s-\xi-u_{x}\right)_{x}, u_{t}\right\rangle_{L^{2}(0, L)}+3 k\left\langle\left(s-\xi-u_{x}\right)_{t},\left(s-\xi-u_{x}\right)\right\rangle_{L^{2}(0, L)} \\
& +\left\langle b s_{x x}-3 k\left(s-\xi-u_{x}\right)-4 \delta s-4 \gamma s_{t}, s_{t}\right\rangle_{L^{2}(0, L)}+b\left\langle s_{x t}, s_{x}\right\rangle_{L^{2}(0, L)} \\
& +4 \gamma\left\langle s_{t}, s\right\rangle_{L^{2}(0, L)}+3\left\langle\theta_{x x}-\nu q_{x}-\mu \xi_{x t}, \theta\right\rangle_{L^{2}(0, L)}-3\left\langle q+\nu \theta_{x}, q\right\rangle_{L^{2}(0, L)} \\
& +3\left\langle b \xi_{x x}+k\left(s-\xi-u_{x}\right)-\beta \xi_{t}-\mu \theta_{x}, \xi_{t}\right\rangle_{L^{2}(0, L)}+3 b\left\langle\xi_{x t}, \xi_{x}\right\rangle_{L^{2}(0, L)} .
\end{aligned}
$$

Integrating by parts on $(0, L)$ and simplifying the terms yield (2.11). 
We now consider the following result.

Proposition 2.2. Let $\varrho(\mathcal{A})$ be the resolvent set of the operator $\mathcal{A}$. We have that $0 \in \varrho(\mathcal{A})$.

Proof. For $F=\left(f^{1}, f^{2}, f^{3}, f^{4}, f^{5}, f^{6}, f^{7}, f^{8}\right)^{T} \in \mathcal{H}$, we must show that there exists a unique $U=$ $\left(u, u_{t}, \xi, \xi_{t}, s, s_{t}, \theta, q\right)^{T}$ in $\mathcal{D}(\mathcal{A})$ such that $\mathcal{A} U=F$, that is,

$$
\begin{aligned}
u_{t} & =f^{1} \quad \text { in } \quad H_{0}^{1}(0, L), \\
\left(s-\xi-u_{x}\right)_{x} & =-\frac{\rho_{1}}{k} f^{2} \quad \text { in } \quad L^{2}(0, L), \\
\xi_{t} & =f^{3} \quad \text { in } \quad H_{0}^{1}(0, L), \\
b \xi_{x x}+k\left(s-\xi-u_{x}\right)-\beta \xi_{t}-\mu \theta_{x} & =\rho_{2} f^{4} \quad \text { in } \quad L^{2}(0, L), \\
s_{t} & =f^{5} \quad \text { in } \quad H_{0}^{1}(0, L), \\
b s_{x x}-3 k\left(s-\xi-u_{x}\right)-4 \delta s-4 \gamma s_{t} & =\rho_{2} f^{6} \quad \text { in } \quad L^{2}(0, L), \\
\theta_{x x}-\nu q_{x}-\mu \xi_{x t} & =f^{7} \quad \text { in } \quad H_{0}^{1}(0, L), \\
-q-\nu \theta_{x} & =f^{8} \quad \text { in } \quad H_{0}^{1}(0, L) .
\end{aligned}
$$

Replacing (2.14) in (2.15), (2.16) in (2.17) and (2.14) into (2.18) we obtain

$$
\begin{aligned}
\left(s-\xi-u_{x}\right)_{x} & =-\frac{\rho_{1}}{k} f^{2} \quad \text { in } \quad L^{2}(0, L), \\
b \xi_{x x}+k\left(s-\xi-u_{x}\right)-\mu \theta_{x} & =\beta f^{3}+\rho_{2} f^{4} \quad \text { in } \quad L^{2}(0, L), \\
b s_{x x}-3 k\left(s-\xi-u_{x}\right)-4 \delta s & =4 \gamma f^{5}+\rho_{2} f^{6} \quad \text { in } \quad L^{2}(0, L), \\
\theta_{x x}-\nu q_{x} & =\mu f_{x}^{3}+f^{7} \quad \text { in } \quad L^{2}(0, L), \\
-q-\nu \theta_{x} & =f^{8} \quad \text { in } \quad H_{0}^{1}(0, L) .
\end{aligned}
$$

Using boundary conditions (2.6) and (2.20) we have

$$
-\frac{\rho_{1}}{k} \int_{0}^{x} f^{2}(y) d y=s-\xi-u_{x} \quad \text { in } \quad L^{2}(0, L) .
$$

From (2.23) and (2.24) we obtain

$$
\begin{aligned}
-\nu q+\theta_{x} & =\mu \int_{0}^{x} f_{x}^{3}(y) d y+\int_{0}^{x} f^{7}(y) d y \text { in } L^{2}(0, L), \\
\nu q+\nu^{2} \theta_{x} & =-\nu f^{8} \quad \text { in } L^{2}(0, L),
\end{aligned}
$$

then, we get

$$
\theta_{x}=\frac{1}{1+\nu^{2}}\left[\mu \int_{0}^{x} f_{x}^{3}(y) d y+\int_{0}^{x} f^{7}(y) d y-\nu f^{8}\right] \quad \text { in } \quad L^{2}(0, L)
$$

and we can write

$$
\begin{aligned}
b \xi_{x x} & =F^{1} \quad \text { in } \quad L^{2}(0, L), \\
b s_{x x}-4 \delta s & =F^{2} \text { in } L^{2}(0, L), \\
\left(1+\nu^{2}\right) \theta_{x x} & =F^{3} \quad \text { in } \quad L^{2}(0, L),
\end{aligned}
$$


where

$$
\begin{gathered}
F^{1}:=\rho_{1} \int_{0}^{x} f^{2}(y) d y+\frac{\mu}{1+\nu^{2}}\left[\mu \int_{0}^{x} f_{x}^{3}(y) d y+\int_{0}^{x} f^{7}(y) d y-\nu f^{8}\right]+\beta f^{3}+\rho_{2} f^{4}, \\
F^{2}:=-3 \rho_{1} \int_{0}^{x} f^{2}(y) d y+4 \gamma f^{5}+\rho_{2} f^{6} \\
F^{3}:=\mu f_{x}^{3}+f^{7}-\nu f_{x}^{8} .
\end{gathered}
$$

By the standard result of elliptical regularity (see [12], theorem 3.3.3, page 135.), the system (2.27)-(2.29) has a unique solution

$$
\xi, s, \theta \in H^{2}(0, L) \cap H_{0}^{1}(0, L) .
$$

Then we plug $\xi$ and $s$ just obtained by solving (2.27) and (2.28) into (2.20) and we yields a unique solvability of $u \in H^{2}(0, L) \cap H_{0}^{1}(0, L)$. Finally, by the regularity of $u, \xi, s, z, \theta$ and (2.12),(2.14),(2.16),(2.19), we have that $U \in \mathcal{D}(\mathcal{A})$ is a unique solution of $\mathcal{A} U=F$. It is clear from the regularity of the linear elliptic equations that $\|U\|_{\mathcal{H}} \leq K\|F\|_{\mathcal{H}}$, for a positive constant $K$ independent of $U$. So we conclude that 0 belongs to resolvent set of $\mathcal{A}$.

The well-posedness of (1.10) is ensured by the following theorem.

Theorem 2.1. For $U_{0} \in \mathcal{H}$, there exists a unique weak solution $U$ of (2.8) satisfying

$$
U \in C((0, \infty) ; \mathcal{H})
$$

Moreover, if $U_{0} \in D(\mathcal{A})$, then

$$
U \in C((0, \infty) ; D(\mathcal{A})) \cap C^{1}((0, \infty) ; \mathcal{H})
$$

Proof. As $\mathcal{D}(\mathcal{A})$ is dense in $\mathcal{H}, \mathcal{A}$ is dissipative and $0 \in \varrho(\mathcal{A})$, we conclude by the Lumer-Phillips theorem (see [24]) that $\mathcal{A}$ generates a $\mathrm{C}_{0}$-semigroup of contractions $S(t)=e^{t \mathcal{A}}$ on $\mathcal{H}$. From semigroup theory, $U(t)=e^{t \mathcal{A}} U_{0}$ is the unique solution of (2.8) satisfying (2.30) and (2.31).

\section{Exponential Stability}

In this section the first result that we are going to present is about the necessary and sufficient conditions of exponential stability of a $C_{0}$-semigroup on a Hilbert space $\mathcal{H}$. This result was obtained by Gearhart [8] and Huang [11] independently (see also Prüss [25]). The following statement is due to Huang.

Theorem 3.1. Let $\{\mathcal{S}(t)\}_{t \geq 0}$ be a $C_{0}$-semigroup of contractions of a linear operators on Hilbert space $\mathcal{H}$ with infinitesimal generator $\mathcal{A}$. Then $\{\mathcal{S}(t)\}_{t \geq 0}$ is exponentially stable if and only if

$$
\{i \zeta: \zeta \in \mathbb{R}\} \subset \varrho(\mathcal{A})
$$

and

$$
\limsup _{|\zeta| \rightarrow \infty}\left\|(i \zeta I-\mathcal{A})^{-1}\right\|<\infty .
$$

Now we introduce our main theorem. 
Theorem 3.2 (Main Theorem). The $C_{0}$-semigroup of contractions $\mathcal{S}(t)=e^{\mathcal{A} t}$, generated by $\mathcal{A}$, is exponentially stable, that is, there exist positive constants $M$ and $w$ such that

$$
\|S(t)\| \leq M e^{-w t}
$$

Proof. First, by a contradiction argument, we will prove that

$$
\{i \zeta: \zeta \in \mathbb{R}\} \subset \varrho(\mathcal{A})
$$

The proof will be covered by three steps.

(a) Let $I$ be the identity operator. Since 0 is in the resolvent of $\mathcal{A}$, by the contraction mapping theorem, for any real $\zeta$ such that $|\zeta|<\left\|\mathcal{A}^{-1}\right\|^{-1}$, the operator $i \zeta I-\mathcal{A}=\mathcal{A}\left(i \zeta \mathcal{A}^{-1}-I\right)$ is invertible. Moreover, $\left\|(i \zeta I-\mathcal{A})^{-1}\right\|$ is a continuous function of $\zeta$ in the interval $\left(-\left\|\mathcal{A}^{-1}\right\|^{-1},\left\|\mathcal{A}^{-1}\right\|^{-1}\right)$.

(b) If $\sup \left\{(i \zeta I-\mathcal{A})^{-1}:|\zeta|<\left\|\mathcal{A}^{-1}\right\|^{-1}\right\}=M<\infty$, then by using the contraction theorem again, the operator

$$
i \zeta I-\mathcal{A}=\left(i \zeta_{0} I-\mathcal{A}\right)\left(I+i\left(\zeta-\zeta_{0}\right)\left(i \zeta_{0} I-\mathcal{A}\right)^{-1}\right)
$$

is invertible for $\left|\zeta-\zeta_{0}\right|<M^{-1}$. Hence choosing $\zeta_{0}$ close enough to $\left\|\mathcal{A}^{-1}\right\|^{-1}$, the set $\left\{\zeta:|\zeta|<\left\|\mathcal{A}^{-1}\right\|^{-1}+M^{-1}\right\}$ is contained in the resolvent set of $\mathcal{A}$ and $\left\|(i \zeta I-\mathcal{A})^{-1}\right\|$ is a continuous function of $\zeta$ in the interval $\left(-\left\|\mathcal{A}^{-1}\right\|^{-1}-M^{-1},\left\|\mathcal{A}^{-1}\right\|^{-1}+M^{-1}\right)$.

(c) Suppose that the statement of this theorem is not true. Then, there exists a real number $\omega \neq 0$ with $\left\|\mathcal{A}^{-1}\right\|^{-1} \leq|\omega|<\infty$ satisfying that the set $\{i \zeta:|\zeta|<|\omega|\}$ is in the resolvent of $\mathcal{A}$ and $\sup \left\{\left\|(i \zeta I-\mathcal{A})^{-1}\right\|:|\zeta|<|\omega|\right\}=\infty$. In this situation we can find a sequence of real numbers $\zeta_{n} \in \mathbb{R}$ with $\zeta_{n} \rightarrow \omega,\left|\zeta_{n}\right|<|\omega|$ and a sequence of complex vector functions $U_{n} \in \mathcal{D}(\mathcal{A})$ with $\left\|U_{n}\right\|=1$ such that $\left\|\left(i \zeta_{n} I-\mathcal{A}\right) U_{n}\right\| \rightarrow 0$, as $n \rightarrow \infty$.

But, $\left(i \zeta_{n} I-\mathcal{A}\right) U_{n}$ is given by

$$
i \zeta_{n}\left(\begin{array}{c}
u_{n} \\
u_{n, t} \\
\xi_{n} \\
\xi_{n, t} \\
s_{n} \\
s_{n, t} \\
\theta_{n} \\
q_{n}
\end{array}\right)-\left(\begin{array}{c}
u_{n, t} \\
-\frac{k}{\rho_{1}}\left(s_{n}-\xi_{n}-u_{n, x}\right)_{x} \\
\xi_{n, t} \\
\frac{1}{\rho_{2}}\left[b \xi_{n, x x}+k\left(s_{n}-\xi_{n}-u_{n, x}\right)-\beta \xi_{n, t}-\mu \theta_{n, x}\right] \\
s_{n, t} \\
\frac{1}{\rho_{2}}\left[b s_{n, x x}-3 k\left(s_{n}-\xi_{n}-u_{n, x}\right)-4 \delta s_{n}-4 \gamma s_{n, t}\right] \\
\theta_{n, x x}-\nu q_{n, x}-\mu \xi_{n, x t} \\
-q_{n}-\nu \theta_{n, x}
\end{array}\right)
$$


then we have

$$
\begin{aligned}
i \zeta_{n} u_{n}-u_{n, t} & \longrightarrow 0, \\
i \zeta_{n} u_{n, t}+\frac{k}{\rho_{1}}\left(s_{n}-\xi_{n}-u_{n, x}\right)_{x} & \longrightarrow 0, \\
i \zeta_{n} \xi_{n}-\xi_{n, t} & \longrightarrow 0, \\
i \zeta_{n} \xi_{n, t}-\frac{1}{\rho_{2}}\left[b \xi_{n, x x}+k\left(s_{n}-\xi_{n}-u_{n, x}\right)-\beta \xi_{n, t}-\mu \theta_{n, x}\right] & \longrightarrow 0, \\
i \zeta_{n} s_{n}-s_{n, t} & \longrightarrow 0, \\
i \zeta_{n} s_{n, t}-\frac{1}{\rho_{2}}\left[b s_{n, x x}-3 k\left(s_{n}-\xi_{n}-u_{n, x}\right)-4 \delta s_{n}-4 \gamma s_{n, t}\right] & \longrightarrow 0, \\
i \zeta_{n} \theta_{n}-\theta_{n, x x}+\nu q_{n, x}+\mu \xi_{n, x t} & \longrightarrow 0, \\
i \zeta_{n} q_{n}+q_{n}+\nu \theta_{n, x} & \longrightarrow 0 .
\end{aligned}
$$

Taking the inner product of $\left(i \zeta_{n} I-\mathcal{A}\right) U_{n}$ with $U_{n}$ in $\mathcal{H}$ we obtain

$$
\left\langle\left(i \zeta_{n} I-\mathcal{A}\right) U_{n}, U_{n}\right\rangle_{\mathcal{H}}=i\left\langle\zeta_{n} U_{n}, U_{n}\right\rangle_{\mathcal{H}}-\left\langle\mathcal{A} U_{n}, U_{n}\right\rangle_{\mathcal{H}}
$$

Taking the real part and using (2.11) after apply Poincaré inequality we obtain

$$
\begin{array}{r}
4 \gamma \int_{0}^{L}\left|s_{n, t}\right|^{2} d x+\frac{3}{C_{P}} \int_{0}^{L}\left|\theta_{n}\right|^{2} d x+3 \int_{0}^{L}\left|q_{n}\right|^{2} d x+3 \beta \int_{0}^{L}\left|\xi_{n, t}\right|^{2} d x \\
\leq \operatorname{Re}\left\langle\left(i \zeta_{n} I-\mathcal{A}\right) U_{n}, U_{n}\right\rangle_{\mathcal{H}} .
\end{array}
$$

Noting that $\left(U_{n}\right)_{n \in \mathbb{N}}$ is bounded and $\left(i \zeta_{n} I-\mathcal{A}\right) U_{n} \rightarrow 0$ we obtain

$$
\begin{aligned}
4 \gamma \int_{0}^{L}\left|s_{n, t}\right|^{2} d x+\frac{3}{C_{P}} \int_{0}^{L}\left|\theta_{n}\right|^{2} d x & +3 \int_{0}^{L}\left|q_{n}\right|^{2} d x+3 \beta \int_{0}^{L}\left|\xi_{n, t}\right|^{2} d x \\
& \leq \operatorname{Re}\left\langle\left(i \zeta_{n} I-\mathcal{A}\right) U_{n}, U_{n}\right\rangle_{\mathcal{H}} \longrightarrow 0 .
\end{aligned}
$$

It follows that

$$
\begin{array}{ccc}
s_{n, t} \rightarrow 0 & \text { as } & n \rightarrow \infty, \\
\theta_{n} \rightarrow 0 & \text { as } & n \rightarrow \infty, \\
q_{n} \rightarrow 0 & \text { as } & n \rightarrow \infty, \\
\xi_{n, t} \rightarrow 0 & \text { as } & n \rightarrow \infty .
\end{array}
$$

From (3.3), (3.5), (3.9) and (3.12) we obtain

$$
\begin{aligned}
& \xi_{n} \rightarrow 0 \quad \text { as } \quad n \rightarrow \infty, \\
& s_{n} \rightarrow 0 \quad \text { as } \quad n \rightarrow \infty .
\end{aligned}
$$

Since

$$
\left\|\xi_{n}\right\|_{H_{0}^{1}(0, L)}^{2}=\left|\xi_{n}\right|_{L^{2}(0, L)}^{2}+\left|\xi_{n, x}\right|_{L^{2}(0, L)}^{2}
$$

we get

$$
\xi_{n, x} \rightarrow 0 \quad \text { as } \quad n \rightarrow \infty
$$


Similarly, we have that

$$
\theta_{n, x} \rightarrow 0 \quad \text { as } \quad n \rightarrow \infty
$$

Taking the inner product of the expressions (3.2) and (3.4) with $\rho_{1} u_{n}$ and $-\rho_{2} \psi_{n}$, respectively, adding the results and taking the real part, we obtain

$$
\begin{aligned}
& k \operatorname{Re}\left(\left\|s_{n}-\xi_{n}-u_{n, x}\right\|_{L^{2}(0, L)}^{2}\right)-b\left\langle\xi_{n, x}, \psi_{n, x}\right\rangle_{L^{2}(0, L)} \\
& -\beta\left\langle\xi_{n, t}, \psi_{n}\right\rangle_{L^{2}(0, L)}-\mu\left\langle\theta_{n, x}, \psi_{n}\right\rangle_{L^{2}(0, L)} \longrightarrow 0 .
\end{aligned}
$$

Thus, it follows that

$$
\begin{aligned}
& k \operatorname{Re}\left(\left\|s_{n}-\xi_{n}-u_{n, x}\right\|_{L^{2}(0, L)}^{2}\right)+b\left\langle\left|\xi_{n, x}\right|,\left|\psi_{n, x}\right|\right\rangle_{L^{2}(0, L)} \\
& +\beta\left\langle\left|\xi_{n, t}\right|,\left|\psi_{n}\right|\right\rangle_{L^{2}(0, L)}+\mu\left\langle\left|\theta_{n, x}\right|,\left|\psi_{n}\right|\right\rangle_{L^{2}(0, L)} \longrightarrow 0
\end{aligned}
$$

and by (3.12), (3.15) and (3.16), we have

$$
s_{n}-\xi_{n}-u_{n, x} \rightarrow 0 \quad \text { as } n \rightarrow \infty,
$$

then as

$$
\left\|s_{n}-\xi_{n}-u_{n, x}\right\|_{H_{0}^{1}(0, L)}^{2}=\left|s_{n}-\xi_{n}-u_{n, x}\right|_{L^{2}(0, L)}^{2}+\left|\left(s_{n}-\xi_{n}-u_{n, x}\right)_{x}\right|_{L^{2}(0, L)}^{2},
$$

we obtain

$$
\left(s_{n}-\xi_{n}-u_{n, x}\right)_{x} \rightarrow 0 \quad \text { as } \quad n \rightarrow \infty .
$$

From (3.2) and (3.18), we get

$$
u_{n, t} \rightarrow 0 \quad \text { as } \quad n \rightarrow \infty
$$

Finally, using (3.1) and (3.19), we obtain

$$
u_{n} \rightarrow 0 \quad \text { as } \quad n \rightarrow \infty
$$

Hence from (3.9)-(3.12), (3.13), (3.14), (3.19) and (3.20) contradict $\left\|U_{n}\right\|=1$, then it is proved that

$$
\{i \zeta: \zeta \in \mathbb{R}\} \subset \varrho(\mathcal{A})
$$

Again, by a contradiction argument we will prove the

$$
\limsup _{|\zeta| \rightarrow \infty}\left\|(i \zeta I-\mathcal{A})^{-1}\right\|<\infty
$$

If (3.21) is not true, then there exists a sequence $\left(V_{n}\right)_{n \in \mathbb{N}}$ such that

$$
\frac{\left\|\left(\lambda_{n} I-\mathcal{A}\right)^{-1} V_{n}\right\|}{\left\|V_{n}\right\|} \geq n, \quad \text { where } \quad \lambda_{n}=i \zeta_{n} .
$$

Hence $\left\|\left(\lambda_{n} I-\mathcal{A}\right)^{-1} V_{n}\right\| \geq n\left\|V_{n}\right\|$. Since $\lambda_{n} \in \varrho(\mathcal{A})$ it follows that there exists a unique sequence $\left(U_{n}\right)_{n \in \mathbb{N}}$ such that $\lambda_{n} U_{n}-\mathcal{A} U_{n}=V_{n}$ with $\left\|U_{n}\right\|=1$ and then

$$
U_{n}=\left(\lambda_{n} I-\mathcal{A}\right)^{-1} V_{n}
$$


with

$$
\left\|U_{n}\right\| \geq n\left\|\lambda_{n} U_{n}-\mathcal{A} U_{n}\right\|
$$

We define $F_{n}=\lambda_{n} U_{n}-\mathcal{A} U_{n}$ and we have

$$
\left\|F_{n}\right\| \leq \frac{1}{n}
$$

where it is follows that $F_{n} \rightarrow 0$ strong in $\mathcal{H}$ as $n \rightarrow \infty$.

Now taking the inner product of $F_{n}$ with $U_{n}$ we have

$$
\lambda_{n}\left\langle U_{n}, U_{n}\right\rangle_{\mathcal{H}}-\left\langle\mathcal{A} U_{n}, U_{n}\right\rangle_{\mathcal{H}}=\left\langle F_{n}, U_{n}\right\rangle_{\mathcal{H}}, \quad \lambda_{n}=i \zeta_{n} .
$$

Taking the real part of (3.22) we have

$$
\operatorname{Re}\left\langle F_{n}, U_{n}\right\rangle_{\mathcal{H}}=-\left\langle\mathcal{A} U_{n}, U_{n}\right\rangle_{\mathcal{H}}
$$

Using that $\left\|U_{n}\right\|$ is bounded, $F_{n} \rightarrow 0$ and (2.11), we have

$$
\begin{array}{r}
4 \gamma \int_{0}^{L}\left|s_{n, t}\right|^{2} d x+\frac{3}{C_{P}} \int_{0}^{L}\left|\theta_{n}\right|^{2} d x+3 \int_{0}^{L}\left|q_{n}\right|^{2} d x+3 \beta \int_{0}^{L}\left|\xi_{n, t}\right|^{2} d x \\
\leq \operatorname{Re}\left\langle F_{n}, U_{n}\right\rangle_{\mathcal{H}} \longrightarrow 0 .
\end{array}
$$

Thus

$$
\begin{array}{ccc}
s_{n, t} \rightarrow 0 & \text { as } & n \rightarrow \infty, \\
\theta_{n} \rightarrow 0 & \text { as } & n \rightarrow \infty, \\
q_{n} \rightarrow 0 & \text { as } & n \rightarrow \infty, \\
\xi_{n, t} \rightarrow 0 & \text { as } & n \rightarrow \infty .
\end{array}
$$

From $F_{n}=\lambda_{n} U_{n}-\mathcal{A} U_{n}$ we have

$$
\begin{aligned}
\lambda_{n} u_{n}-u_{n, t} & =f_{1 n}, \\
\lambda_{n} u_{n, t}+\frac{k}{\rho_{1}}\left(s_{n}-\xi_{n}-u_{n, x}\right)_{x} & =f_{2 n}, \\
\lambda_{n} \xi_{n}-\xi_{n, t} & =f_{3 n}, \\
\lambda_{n} \xi_{n, t}-\frac{1}{\rho_{2}}\left[b \xi_{n, x x}+k\left(s_{n}-\xi_{n}-u_{n, x}\right)-\beta \xi_{n, t}-\mu \theta_{n, x}\right] & =f_{4 n}, \\
\lambda_{n} s_{n}-s_{n, t} & =f_{5 n}, \\
\lambda_{n} s_{n, t}-\frac{1}{\rho_{2}}\left[b s_{n, x x}-3 k\left(s_{n}-\xi_{n}-u_{n, x}\right)-4 \delta s_{n}-4 \gamma s_{n, t}\right] & =f_{6 n}, \\
\lambda_{n} \theta_{n}-\theta_{n, x x}+\nu q_{n, x}+\mu \xi_{n, x t} & =f_{7 n}, \\
\lambda_{n} q_{n}+q_{n}+\nu \theta_{n, x} & =f_{8 n},
\end{aligned}
$$

where

$$
f_{\text {in }} \rightarrow 0 \quad \text { in } \quad L^{2}(0, L), \quad \text { for } \quad i=1, \ldots, 8 \text {. }
$$


From (3.23), (3.26), (3.29), (3.31) and (3.35), we obtain

$$
\begin{aligned}
& \xi_{n} \rightarrow 0 \quad \text { as } n \rightarrow \infty, \\
& s_{n} \rightarrow 0 \quad \text { as } \quad n \rightarrow \infty .
\end{aligned}
$$

For previously used arguments we have to

$$
\begin{aligned}
& \xi_{n, x} \rightarrow 0 \quad \text { as } \quad n \rightarrow \infty \\
& \theta_{n, x} \rightarrow 0 \quad \text { as } \quad n \rightarrow \infty .
\end{aligned}
$$

Taking the inner product of the expressions (3.28) and (3.30) with $\rho_{1} u_{n}$ and $\rho_{2} \psi_{n}$, respectively, adding the results and taking the real part, we obtain

$$
\begin{aligned}
k \operatorname{Re}\left(\left\|s_{n}-\xi_{n}-u_{n, x}\right\|_{L^{2}(0, L)}^{2}\right)= & \rho_{1}\left\langle f_{2, n}, u_{n}\right\rangle_{L^{2}(0, L)}-\rho_{2}\left\langle f_{4, n}, \psi_{n}\right\rangle_{L^{2}(0, L)} \\
& +b\left\langle\xi_{n, x}, \psi_{n, x}\right\rangle_{L^{2}(0, L)}+\beta\left\langle\xi_{n, t}, \psi_{n}\right\rangle_{L^{2}(0, L)} \\
& +\mu\left\langle\theta_{n, x}, \psi_{n}\right\rangle_{L^{2}(0, L)} .
\end{aligned}
$$

Thus, it follows that

$$
\begin{aligned}
k \operatorname{Re}\left(\left\|s_{n}-\xi_{n}-u_{n, x}\right\|_{L^{2}(0, L)}^{2}\right)= & \rho_{1}\left\langle\left|f_{2, n}\right|,\left|u_{n}\right|\right\rangle_{L^{2}(0, L)}+\rho_{2}\left\langle\left|f_{4, n}\right|,\left|\psi_{n}\right|\right\rangle_{L^{2}(0, L)} \\
& +b\left\langle\left|\xi_{n, x}\right|,\left|\psi_{n, x}\right|\right\rangle_{L^{2}(0, L)}+\beta\left\langle\left|\xi_{n, t}\right|,\left|\psi_{n}\right|\right\rangle_{L^{2}(0, L)} \\
& +\mu\left\langle\left|\theta_{n, x}\right|,\left|\psi_{n}\right|\right\rangle_{L^{2}(0, L)},
\end{aligned}
$$

and by (3.26), (3.35), (3.38) and (3.39), we have

$$
s_{n}-\xi_{n}-u_{n, x} \rightarrow 0 \quad \text { as } \quad n \rightarrow \infty,
$$

so

$$
\left(s_{n}-\xi_{n}-u_{n, x}\right)_{x} \rightarrow 0 \quad \text { as } \quad n \rightarrow \infty .
$$

From (3.28), (3.35) and (3.41), we get

$$
u_{n, t} \rightarrow 0 \quad \text { as } \quad n \rightarrow \infty \text {. }
$$

Finally, by (3.27), (3.35) and (3.42), we obtain

$$
u_{n} \rightarrow 0 \quad \text { as } \quad n \rightarrow \infty \text {. }
$$

Then from (3.23)-(3.26), (3.36), (3.37), (3.42) and (3.43) we conclude that $U_{n} \rightarrow 0$ that is a contradiction with $\left\|U_{n}\right\|=1$. The proof of the theorem is complete.

Acknowledgments. This work is supported in part by Brazilian-French Network in Mathematics. The second author was partially supported by project FONDECYT/1191137. The third author was partially supported by CAPES (Brasil). The fourth author wishes to thank the financial support of the Laboratoire de Mathématiques Appliquées, Université de Pau et des Pays de l'Adour and CNRS (France). He is also supported in part by ALV'2012, Universidade Federal do Rio de Janeiro (Brazil). 


\section{REFERENCES}

[1] B. A. H. Abbas and J. Thomas, The second frequency spectrum of Timoshenko beams, J. Sound Vib. 51 (1977), $123-137$.

[2] R. A. Adams, Sobolev Spaces, Academic Press, New York, 1973.

[3] D. S. Almeida Júnior, J. E. Muñoz Rivera and M. L. Santos, The stability number of the Timoshenko system with second sound, J.Differ.Equ. 253 (2012), 2715-2733.

[4] M. S. Alves, P. Gamboa, G. C. Gorain, A. Rambaud and O. Vera, Asymptotic behavior of a flexible structure with Cattaneo type of thermal effect, Indagationes Mathematicae. 27 (2016), 821-834.

[5] A. Borichev and Y. Tomilov, Optimal polynomial decay of functions and operator semigroups, Math. Ann. 347 (2009), $455-478$.

[6] X-G. Cao, D. Liu and G. Q. Xu, Easy test for stability of laminated beams with structural damping and boundary feedback controls, J. Dynamical Control Syst. 13 (2007), 313-336.

[7] B. Feng, T. F. Ma, R. N. Monteiro and C. A. Raposo, Dynamics of Laminated Timoshenko Beams, J Dyn Diff Equat. 30 (2018), $1489-1507$.

[8] L. M. Gearhart, Spectral theory for contraction semigroups on Hilbert spaces, Trans. Amer. Math. Soc. 236 (1978), $385-394$.

[9] S. W. Hansen, In Control and Estimation of Distributed Parameter Systems: Non-linear Phenomena, International Series of Numerical Analysis. 118 (1994), 143-170.

[10] S. W. Hansen and R. Spies, Structural damping in a laminated beams due to interfacial slip, J. Sound Vib. 204 (1997), $183-202$.

[11] F. L. Huang, Characteristic condition for exponential stability of linear dynamical systems in Hilbert spaces, Ann. Diff. Eqns. 1 (1985), $43-56$.

[12] S. Kesavan, Topics in Functional Analysis and Applications, John Wilye \& Sons, New York, 1989.

[13] V. Komornik, Exact controllability and stabilization. The multiplier method, RAM: Research in Applied Mathematics, Paris, 1994.

[14] V. Kormonik and E. Zuazua, A direct method for the boundary stabilization of the wave equation, J. Math. Pures Appl. 69 (1990), 33-54.

[15] A. Lo and N. E. Tatar, Stabilization of laminated beams with interfacial slip. Electron. J. Diff. Eqs. 129 (2015), 1-14.

[16] A. Lo and N. E. Tatar, Exponential stabilization of a structure with interfacial slip, Discrete Contin. Dyn. Syst. 36 (2016), 6285-6306.

[17] Z. Liu and S. Zheng, Semigroups associated with dissipative systems, Chapman \& Hall/CRC, New York, 1999.

[18] S. A. Messaoudi, M. Pokojovy and B. Said-Houari, Nonlinear damped Timoshenko systems with second sound-global existence and exponential stability, Math. Meth. Appl. Sci. 32 (2009), 505-534.

[19] S. A. Messaoudi and B. Said-Houari, Exponential stability in one-dimensional non-linear thermoelasticity with second sound, Math. Meth. Appl. Sci. 28 (2005), 205-232.

[20] J. E. Muñoz Rivera, Energy decay rates in linear thermoelasticity, Funkcial EKVAC. 35 (1992), 9-30.

[21] J. E. Muñoz Rivera and R. Racke, Timoshenko systems with indefinite damping, J. Math. Anal. Appl. 341 (2008), $1068-1083$.

[22] J. E. Muñoz Rivera and R. Racke, Mildly dissipative nonlinear Timoshenko systems—global existence and exponential stability, J. Math. Anal. Appl. 276 (2002), 248-278.

[23] M. Nakao, On the decay of solutions of some nonlinear dissipative wave equations, Math. Z. Berlin. 193 (1986), $227-234$.

[24] A. Pazy, Semigroups of Linear Operators and Applications to Partial Differential Equations, Springer- Verlag, New York, 1983.

[25] J. Prüss, On the spectrum of $C_{0}$-semigroups, Trans. Amer. Math. Soc. 284 (1984), 847-857.

[26] R. Racke, Thermoelasticity with second sound - exponential stability in linear and non-linear 1 - d, Math. Meth. Appl. Sci. 25 (2002), 409-441.

[27] C. A. Raposo, Exponential stability for a structure with interfacial slip and frictional damping, Appl. Math. Lett. 53 (2016), 85-91.

[28] C. A. Raposo, O. Vera Villagran, J. E. Munoz Rivera and M. S. Alves, Hybrid laminated Timoshenko beam, J. Math. Phys. 58 (2017), 101512.

[29] C. A. Raposo, J. Ferreira, M. L. Santos, N. N. Castro, Exponential stabilization for the Timoshenko system with two weak dampings, Appl. Math. Lett. 18 (2005), 535-541.

[30] B. Said-Houari and A. Kasimov, Decay property of Timoshenko system in thermoelasticity, Math. Methods Appl. Sci. 35 (2012), 314-333. 
[31] H. D. F. Sare and R. Racke, On the stability of damped Timoshenko systems: Cattaneo versus Fourier law, Arch. Rational Mech. Anal. 194 (2009), 221-251.

[32] M. A. Tarabek, On the existence of smooth solutions in one-dimensional nonlinear thermoelasticity with second sound, Quarterly of Applied Mathematics. 50 (1992), 727-742.

[33] J. M. Wang, G. Q. Xu and S. P. Yung, Exponential stabilization of laminated beams with structural damping and boundary feedback controls, SIAM J. Control Optim. 44 (2005), 1575-1597. 